\title{
Negocios con TIC y valores: \\ Una experiencia curricular en la Educación Superior Business with ICT and values: A curricular experience in the Superior Education
}

\author{
Alba Zulay Cárdenas Escobar ${ }^{1}$, Gilma Mestre de Mogollón ${ }^{2}$ \\ email acardenas@unitecnologica.edu.co,email gmestre@unitecnologica.edu.co \\ ${ }^{1}$ Facultad de Economía y Negocios \\ Universidad Tecnológica de Bolívar \\ Cartagena de Indias, Colombia \\ ${ }^{2}$ Facultad de Educación \\ Universidad Tecnológica de Bolívar \\ Cartagena de Indias, Colombia
}

\begin{abstract}
Resumen- Este documento tiene por objeto presentar los resultados de la implementación del piloto del rediseño curricular efectuado al curso de Informática Aplicada, curso obligatorio en el primer semestre del pensum académico de todos los programas de la Facultad de Economía y Negocios de la Universidad Tecnológica de Bolívar, y compartir con la comunidad académica la pertinencia y el impacto de los cambios implementados, al propiciar el desarrollo de competencias como el aprendizaje autónomo, el trabajo colaborativo y cooperativo y fomentar el compromiso ético en la población estudiantil intervenida, trascendiendo el ámbito académico al profesional, personal y familiar, usando como mediación las Tecnologías de la Información y la Comunicación - TIC.
\end{abstract}

Palabras clave: TIC, Valores Ciudadanos, Rediseño Curricular, MOOC, Redes Sociales.

Abstract- This document shows the results of the pilot's implementation of the curricular redesign carried out to the course of Applied Computing, a compulsory course in the first semester of the academic curriculum of all the programs of the Faculty of Economy and Business of Tecnológica de Bolívar University, and share with the academic community the relevance and impact of the implemented changes, by fostering the development of competences such as autonomous learning, collaborative and cooperative work and fostering ethical commitment in the student population intervened, transcending the academic field to the professional, personal and family, using as mediation the Information and Communication Techologies - ICT.

Keywords: ICT, Citizen Values, Curricular Redesing, MOOC, Social Networks.

\section{INTRODUCCIÓN}

Este trabajo presenta la experiencia del rediseño curricular del curso de Informática Aplicada presente en el pensum académico de todos los programas de la Facultad de Economía y Negocios de la Universidad Tecnológica de Bolívar (Cartagena - Colombia), iniciativa que surge de la necesidad de vincular el conocimiento a una “comprensión de las situaciones sociales, de las acciones humanas, y consiguientemente de las cuestiones de valor controvertidas que le son consustanciales” (Torres, 1998, p. 3). Se trata de reinventar un curso que se venía desarrollando con una orientación tradicional, netamente técnica y procedimental, en bajo grado articulado con los demás cursos del pensum educativo, para pasar a un enfoque humanista que sin dejar a un lado las herramientas tecnológicas, las vincula con los intereses y necesidades de los estudiantes desde el punto de vista educativo. Esto es, encontrar en sus contenidos otras formas de relación con la ética, los valores, el ámbito laboral y el académico con otros cursos, en un enfoque interdisciplinar y de integración curricular.

En otras palabras, una propuesta curricular crítica, que por lo tanto, supone modificar la visión con la que se venía desarrollando el curso, para proponer una acción educativa más humanista que supone pasar de una planificación lineal, reproductiva y rutinaria, a una mirada innovadora, quizá retadora, en cuanto si interesaría o no a los estudiantes y que a su vez lograra las metas propuestas en el curso.

En ese orden, las estrategias empleadas, tales como: inscribirse como estudiante en un curso masivo abierto en línea gratuito (MOOC, por sus siglas en inglés) libre en una plataforma educativa con reconocimiento por su calidad; mantener un foro en un aula virtual, siendo el curso presencial, para analizar el uso adecuado de las Tecnologías de la Información y la Comunicación ( TIC), y con ello el respeto a las citas de autor; o elaborar exposiciones orales luego de aprender a utilizar herramientas para tal fin, se convirtieron en verdaderos escenarios educativos para el aprendizaje significativo.

\section{CONTEXTO}

El diseño curricular de un curso, como es el caso que aquí se presenta, dirigido a estudiantes de carreras universitarias, implica tener en cuenta el perfil del profesional que se espera egrese del mismo, y así responder lo más aproximadamente posible a los intereses, expectativas y necesidades de un mundo que en el presente siglo, ha de atender a los fenómenos de globalización, de competitividad, internacionalización, trabajo en redes, entre otros.

Se está entonces frente a uno de los casos planteados por Zabalza, M.(1997) para efectos de rediseñar un Programa, entre cuyas razones el autor "considera que el Programa, 
precisa de una reformulación que suponga una mejor acomodación a las condiciones de la situación” ( p. 5) actual.

En ese sentido, a partir de una perspectiva de diseño curricular en el que además de tener en cuenta el contexto social, laboral y profesional del estudiante, se atiende al compromiso de la institución educativa con el Sistema Educativo Colombiano, en el desarrollo de las capacidades crítica, analítica y reflexiva, que aporte al avance científico y tecnológico, es importante relacionarlo con la responsabilidad en la solución de problemáticas económicas y sociales aspectos encomendados por la Ley colombiana de formar con sentido ético, moral y de participación ciudadana. La Ley 749 (2002) en su artículo 15, literalmente demanda por "diseños curriculares con la debida pertinencia social y académica”.

Así, “cada tema, cada contenido, cada concepto debería asumirse no como resultado, sino de manera dinámica como actividad, como vector, como proceso, como interrogante constitutivo del mismo conocimiento científico" (Posner, 2000. p. XXVII).

Es decir, una propuesta curricular flexible, abierta, pertinente, creativa que atiende individualidades y necesidades del contexto, considera que el concepto diseño curricular responde a un conjunto de acciones y experiencias programadas por la institución con sentido de oportunidades de aprendizaje puestas en práctica en el proyecto curricular. Lo cual, conforme lo plantea Stenhouse, citado por Elliot (1983), se trata de responder al derecho del estudiante a un saber que se conecta con el conocimiento del mundo extraescolar ya que trasciende el aula, llega a la familia y al mundo laboral, mediante una acción educativa, dialogante, participativa y colaborativa.

Así, tenemos un docente y estudiantes aprendiendo, consultando, investigando, reorganizando contenidos alrededor de temas de la vida diaria, de los requerimientos de la sociedad actual y de la evolución del conocimiento.

\section{A. Marco conceptual}

El presente trabajo de rediseño del curso de Informática Aplicada, parte de la importancia de mantener un currículo actualizado con pertinencia al contexto, se inscribe en el eje temático de tecnologías emergentes en la formación y el aprendizaje, y en el de aprendizaje autónomo y aprendizaje en redes sociales.

Currículo pertinente para un aprendizaje autónomo. Un currículo es pertinente cuando integra y da respuesta a un cúmulo de necesidades que tiene el estudiante que aspira a formarse como un profesional competitivo para el mundo de hoy.

Esto implica una mayor integración entre la universidad y la sociedad, lo cual conforme lo expresa Malagón Plata (2004, p. 179) “(...) el papel que la universidad cumple en el contexto de la formación social: no se trata simplemente de un papel ideológico (cultural), simbólico, sino de un papel político, social y económico (...)”

Se trata entonces de ver en la propuesta del curso el cumplimiento de una educación para la vida, conforme lo expresa Jacques Délors en su informe a la Unesco (1998): aprender a conocer, aprender a hacer, aprender a vivir juntos y aprender a ser. Lo anterior unido a los siete (7) saberes expresados por Morin (1999) y entre los cuales se encuentra el principio de un conocimiento pertinente, entendido como la reflexión que ha de hacer el docente de su praxis pedagógica para organizar su curso teniendo en cuenta cuáles son esos conocimientos relacionados con los ejes temáticos que le permiten decidir los conocimientos necesarios de aprender, de enseñar, los conocimientos que de acuerdo con la disciplina se convierten en imponderables y por lo tanto, de marcado énfasis, los necesarios para hacer y transformar su ámbito, para relacionarse con sus semejantes y por consiguiente para ser un ciudadano del planeta con todo lo que ello significa.

"En el siglo XXI la educación superior no sólo tendrá que ser pertinente, sino que además esa pertinencia será juzgada en términos de productos, de la contribución que la educación superior haga al desempeño de la economía nacional $\mathrm{y}$, a través de ello, del mejoramiento de las condiciones de vida" (Gibbons, M., 1998). Así, los procesos de formación en una propuesta curricular tendrán pertinencia para el siglo actual, en la medida en que todo lo que se aprenda contribuya a la formación de una persona integral, al desempeño profesional, y a la transformación humana y de la sociedad.

De acuerdo con lo anterior, la propuesta curricular ha de incluir aspectos de innovación a los procesos pedagógicos y didácticos orientados a la producción de un conocimiento holístico y con sentido humano. Así, en el momento actual, el uso de las TIC se convierte en herramienta necesaria en el ambiente educativo para interesar al estudiante y ampliarle la visión competitiva de la sociedad actual. Estas a su vez contribuyen en la construcción de tejido social al organizar el curso para un desarrollo en equipo, con trabajo colaborativo y cooperativo que fortalecen el aprendizaje autónomo.

Tecnologías emergentes para formación y el aprendizaje. La dinámica de inmersión de las TIC, en los diferentes ámbitos, incluido el educativo, en el cual se ha creado un nuevo medio de relación entre estudiantes, docente y estudiantes, ha ampliado los tiempos de contacto favoreciendo el aprendizaje, superando el escenario del aula o institucional y llegando hasta la familia misma. Por su parte, la globalización con fenómenos como la internacionalización del currículo ha traído consigo posibilidades inmensas de apropiar aprendizajes mediante el acceso a plataformas virtuales que llevan a los estudiantes a otros escenarios de aprendizaje.

Las TIC ofrecen la posibilidad de crear entornos nuevos de relación, y como tales, deben de ser tratados de forma distinta para extraer de ellos el máximo de su potencial. La riqueza de estos nuevos entornos, todavía en fase de exploración, es enorme y su poder reside en nuestra capacidad de saber usarlos al máximo de sus posibilidades. Debemos cambiar de hábitos, ser creativos, para rendir en este nuevo medio mientras podamos hacerlo. En la generalización del aprendizaje para el uso, y para el saber estar y saber participar en ese medio, está la clave del éxito.

La educación no puede ser ajena al potencial que los nuevos espacios de relación virtual aportan. Ante la rapidez de la evolución tecnológica, ahora más que nunca, la educación debe manifestarse claramente y situar la tecnología en el lugar que le corresponde: el de medio eficaz para garantizar la comunicación, la interacción, la información y, también, el aprendizaje. Herramientas como los foros virtuales, permiten una relación de comunicación asincrónica en la que los estudiantes comparten opiniones, saberes, documentos de interés entre sus compañeros, ampliando así las posibilidades 
de aprendizajes y modificando roles en los que era solo el docente quien proponía para pasar a la de un estudiante que lidera y toma las riendas de una conversación, con sus compañeros y el docente mismo.

\section{B. Contexto Disciplinar}

La estructura curricular de los programas de la Facultad de Economía y Negocios de la Universidad Tecnológica de Bolívar corresponde al enfoque curricular crítico, cuya orientación central pretende que estudiantes y docentes construyan en forma conjunta el conocimiento; se enfrenten a los problemas de la realidad objetiva, por lo tanto, para la selección de contenidos en los cursos, es la propia realidad la que suministra los temas y problemas pertinentes para la formación. A su vez, se pretende que los estudiantes sean participantes activos en la construcción de su aprendizaje sobre la base de una adecuada interacción docente - estudiante, estudiante - estudiante y estudiante - contenido de enseñanza.

Siguiendo con la estructura curricular, conviene afirmar que la piedra angular dentro de este paradigma crítico está representada por el interés emancipador del currículo. En ese sentido se resalta que por ejemplo para Habermas (1986), "emancipación significa independencia de todo lo que está fuera del individuo y se trata de un estado de autonomía más que de libertinaje.”; por lo tanto, los currículos de los diferentes cursos en la Facultad de Economía y Negocios de la Universidad Tecnológica de Bolívar tienen dentro de sus propósitos sustantivos el desarrollo de competencias para incorporar el aprendizaje autónomo, brindándole al estudiante las estrategias y métodos necesarios para aprender a aprender durante todo su proceso de formación.

La anterior afirmación se refleja en la composición del currículo, en el cual se observan diferentes áreas y cursos que tienen intensidad horaria diversa. En cada curso se requiere que el estudiante realice actividades independientes e importantes como por ejemplo lectura, realización de talleres individuales y /o grupales, entre otros, que se contemplan bajo la modalidad de trabajo autónomo. Visto desde la normativa del sistema de créditos, se asume que por cada hora de clase que recibe el estudiante, debe trabajar el doble en forma autónoma.

Basados en la concepción de Competencias Genéricas contempladas en el Proyecto Educativo Institucional PEI de la Universidad se entiende que éstas: Son transversales a todos los programas en virtud de la interdisciplinariedad del conocimiento y a la necesidad de complementariedad de las disciplinas del conocimiento. Su apropiación tiene lugar en las experiencias de aprendizaje que se le proveen al estudiante y las que el mismo medio le ofrece. Los diferentes programas de la Facultad de Economía y Negocios de la Universidad Tecnológica de Bolívar orientan sus vivencias académicas hacia el logro de competencias referidas al:

- Compromiso ético y profesional

- Iniciativa y espíritu emprendedor

- Trabajo en equipos disciplinares y multidisciplinares

- Capacidad para trabajar en forma autónoma

- Habilidades para comunicarse eficientemente por diferentes medios y en escenarios de alta exigencia académica

- Conocimiento de temas contemporáneos que le permitan interactuar en ámbitos de la vida nacional e internacional.
- $\quad$ Aprender de manera autónoma y aprender a desaprender

- Aplicar técnicas, destrezas y herramientas modernas propias de su profesión para la práctica de la misma.

- Participar activamente en la solución de problemas y en la proposición de alternativas para el desarrollo local, regional, nacional, internacional y personal. (PEI Universidad Tecnológica de Bolívar, 2005, p 24).

\section{Población Objetivo}

La población objetivo a intervenir e impactar son los estudiantes de primer semestre de la Facultad de Economía y Negocios de la Universidad Tecnológica de Bolívar, matriculados en el curso Informática Aplicada, curso de 3 créditos académicos (cada crédito académico comprende 48 horas de horas de labor académica del estudiante entre trabajo presencial e independiente), 4 horas presenciales de clases semanales desarrolladas en las 16 semanas que tiene un semestre académico, para un total 64 horas presenciales de acompañamiento.

\section{DESCRIPCIÓN}

En el curso de Informática Aplicada ubicado en el primer semestre de la malla curricular o pensum de los cinco programas académicos de la Facultad de Economía y Negocios de la Universidad Tecnológica de Bolívar, introducido en la reforma curricular de 2007, tuvo como propósito en sus inicios, desarrollar competencias y habilidades en el uso de las herramientas de ofimática de Microsoft Office: el procesador de texto - Word, la hoja de presentación - Power Point y la hoja de cálculo electrónica Excel, dado que se evidenció que los estudiantes no recibían esta formación en su educación secundaria.

En el año 2010, las instituciones educativas tanto públicas como privadas, adoptan en su Proyecto Educativo Institucional (PEI), el área de Informática, implementación que ya había sido proyectada en el documento Visión 2019: Educación (MEN, 2006, p 59), y en la Universidad comienzan a observarse cambios en la población de estudiantes de primer semestre que se evidencian en el manejo del Word y del Power Point, básicamente. Por tal razón, en el segundo semestre de 2011 se decide en el consejo de Facultad de Economía y Negocios, modificar el currículo del curso de Informática Aplicada concentrando su contenido en las funciones y herramientas de la hoja de cálculo de Excel, contextualizada a su uso en las matemáticas, economía, finanzas, estadísticas e investigación de mercados.

Producto de haber dirigido un proyecto de Innovación Educativa con cobertura regional y nacional en Colombia durante los años 2014-2016, en el cual se desarrollaron contenidos educativos y se formaron docentes en el uso de herramientas para el desarrollo de contenidos educativos, una de las autoras de esta ponencia propone a las direcciones de programa y a la decanatura de la Facultad, hacer un rediseño curricular del curso y ejecutar un piloto en el primer semestre de 2017, con miras a masificar en el segundo semestre del mismo año, alineado al proceso de reforma curricular que se viene ejecutando en toda la Universidad desde finales del 2015.

El rediseño curricular propuesto se enfoca en fomentar la ética y valores; expresar ideas de manera asertiva tanto de 
forma oral como escrita; el trabajo colaborativo y aprendizaje con otros; el aprendizaje autónomo y autogestionado; la búsqueda, selección y procesamiento de la información en la internet y redes sociales. Todo lo anterior basado en que las TIC son las herramientas esenciales para insertarse en la sociedad del conocimiento. De igual manera, el rediseño busca sensibilizar a los estudiantes sobre las megatendencias globales que en TIC y negocios se vislumbran en las próximas tres décadas, dentro de las cuales cabe mencionar el uso del big data, el cibercrimen y la transformación de las comunicaciones, entre otros (KPMG, 2014, p 23).

El piloto del curso rediseñado se implementó en cuatro grupos que sumaron un total de 110 estudiantes (considerada la muestra de una población total de 200 estudiantes de primer semestre de la Facultad, matriculados en el primer semestre de 2017). Las temáticas y actividades desarrolladas fueron en su orden:

Primeras ocho (8) semanas: los temas abordados se centraron en el conocimiento y uso de herramientas para crear y publicar contenidos teniendo como hilo conductor la ética en los negocios y valores ciudadanos en general. Las actividades que se llevaron a cabo fueron:

Foro sobre herramientas de accesibilidad en celulares inteligentes (smartphones), para evidenciar mecanismo de inclusión social mediados por las TIC.

Taller de elaboración de documentos, presentaciones, hojas de cálculo y formularios con las aplicaciones de Google Drive, para fomentar el trabajo colaborativo y aprendizaje con otros.

Taller de Prezi y PowToon sobre normas APA, énfasis en citación y referenciación, para fomentar el respeto a los derechos de autor.

Taller de Pixton con base en historias y fábulas para fomentar y fortalecer conceptos de ética y valores ciudadanos al tiempo que se desarrolle la habilidad de narrar historias (Storytelling).

El trabajo final de esta primera parte del curso fue un trabajo escrito elaborado con Google Docs; diseño de archivo de presentación elaborado con Power Point (avanzado), Prezi, PowToon y/o Pixton y una exposición oral, en grupo de dos (parejas). Los tres entregables por grupo, giraron alrededor de la temática "Peligros y amenazas en las redes sociales"; a cada grupo se le asignó un tema dentro de doce subtemas, entre los cuales vale la pena mencionar: Grooming, Sexting, Phishing, Malware, Ciberbullying, Apps para contener peligros en las redes, entre otros. Al final de las exposiciones se crearon las redes sociales del grupo en las cuales se publicaron las presentaciones y los talleres desarrollados en las semanas previas.

Segunda parte del curso (8 semanas restantes), se concentraron en el manejo de la hoja de cálculo electrónica Excel para desarrollar habilidades y destrezas para desarrollar bases de datos y modelos genéricos con aplicación futura en cursos de matemáticas, mercados, estadística, economía y finanzas, en últimas en negocios.

Cabe mencionar que como actividad transversal al curso y como una forma de garantizar un uso efectivo de parte de las horas de trabajo independiente del mismo ( teniendo en cuenta la definición de crédito académico, el curso tiene un total de 80 horas de trabajo independiente), desde la primera clase se le presentaron a los estudiantes tres plataformas educativas para desarrollo gratuito de Cursos en línea, masivos y abiertos MOOC (Massive Online Open Courses, por sus siglas en inglés): Coursera, MiriadaX y Ecolearning con el fin de que de manera autónoma eligieran un curso de su interés se inscribieran $\mathrm{y}$ al final del semestre debían presentar la evidencia de terminación exitosa ( pantallazos y emails) y con la cual se asignaría una nota al final del curso.

A partir de la ejecución del piloto, se propone a la decanatura de la facultad modificar el nombre del curso de Informática Aplicada a TIC y Negocios o TIC aplicada a los Negocios.

Como mecanismo inmediato para evaluar el impacto se realizaron entrevistas focales (focus group interviews) con preguntas abiertas a estudiantes que tomaron el curso, con una muestra significativa de cinco estudiantes por cada uno de los cuatro grupos, las preguntas tuvieron como propósito indagar sobre la percepción del curso, su contenido, competencias (saber, hacer y ser - convivir) y pertinencia (aplicación profesional y personal).

\section{Resultados}

Los impactos se presentan en función de las competencias desarrolladas y/ o fortalecidas en los estudiantes: autonomía, creatividad, colaboración y cooperación que se evidencian a través de los productos ( trabajos ) desarrollados y entregados por los estudiantes y subidos a la plataforma de apoyo tecnológico de la Universidad llamada SAVIO y también disponibles de manera abierta en las redes sociales que se crearon del curso en Twitter (TIC\&Negocios @negocios_tic), Facebook (TicyNegocios Fen) e Instagram (TIC\&Negocios tic_y_negocios).

Los resultados de las entrevistas focales (focus group interviews) se presentan a continuación, agrupados en cinco categorías a saber: percepción general del curso; exposiciones orales sobre uso de las redes sociales; plataformas educativas en línea y MOOC; normas APA; herramientas para desarrollo de contenidos, ética y valores, fueron:

Percepción general del curso, con preguntas como qué gustó y qué no gustó. Las respuestas entregadas por los estudiantes se reflejan en expresiones tales como: "Dimensionamos el alcance que tiene la tecnología y su impacto más allá del campo laboral”; otro estudiante manifestó “ Es la materia más integral que hemos dado en todo el semestre: atiende a asuntos en lo personal, académico, laboral, aplicación en la vida diaria”, Otras opiniones fueron: “ la clase en general aporta al bienestar personal”, "todos los cursos deben ir encaminados en todos los sentidos como éste”, "el curso me permitió aplicar las herramientas de accesibilidad de mi celular con mis abuelitos”, “me gustó Prezi”, “ bastante relevante el curso”, “ se debe asignar más tiempo al tema de Excel”, "la profesora debería tener un monitor o asistente”, “ la profe exige lectura previa así que uno no llega tan azul ( significa en blanco, sin idea de referencia) a la clase”. Las anteriores opiniones pueden entenderse como una apreciación bastante favorable al curso por cuanto reconocen su utilidad y aplicación en diferentes contextos, desde el mismo empleo en lo académico, así como en lo laboral y familiar.

En cuanto a las exposiciones orales relacionadas con el uso de redes sociales manifestaron lo siguiente: “Dándole un 
buen uso, aprendimos cómo prevenir peligros" "No solo ver el Facebook para montar foto, sino compartiendo y aportando conocimiento a nuestros amigos [...] no solo ser vanidosos", "las exposiciones sobre los peligros en las redes sociales fueron de mucha actualidad". Este es el reconocimiento a la ampliación de sus saberes en cuanto al uso de las TIC, que les amplío su comprensión de que las redes sociales son más que para usos banales por fuera del contexto académico.

Sobre los cursos libres en las plataformas educativas manifestaron que: "Me decidí por el curso negocios internacionales, en la plataforma de Coursera para aprender más sobre lo que trata mi carrera” otro estudiante "yo elegí en la misma plataforma el curso de Gestión de Personas pues me pareció interesante”, “yo escogí Excel para negocios”. Cabe decir que las evidencias de inscripción y terminación de los cursos permiten decir que los estudiantes fueron más allá de preferencias profesionales en su selección; algunos optaron por cursos para aprender idiomas como el Coreano y el Chino, hasta hobbies como Egiptología y Arte en 3D. Hubo quien anotara que le contó a su hermana, también profesional, acerca de estos cursos y también terminó matriculándose en uno de su interés. Esto demuestra que una propuesta curricular abierta, flexible y participativa, sí trasciende el aula de clase y de la institución para llegar a la familia

Sobre cómo hacer un trabajo con normas APA, un estudiante dijo "Sobrevivo en ese aspecto gracias a este curso que me enseñó a reconocer los derechos de autor, las implicaciones legales de no hacerlo". Otro manifestó "No tenía ni idea de que significaba APA y con la profe aprendí sobre ellas y que usándolas, evita caer en el plagio”. Todo ello nos muestra que los valores éticos y las normas sociales no se aprenden a fuerza de repetirlas o de castigos, sino que pueden estar de manera transversal e interdisciplinar en cualquier curso. Parecería extraño pensar que un curso del área de los negocios pudiera verse relacionado con los valores, pero es así. La educación para el siglo actual ha de ser integral y toda acción dirigida a las personas debe tener su enfoque humanístico.

Herramientas para el desarrollo de contenidos y la ética y valores. Un estudiante manifestó "en el taller de Pixton cada uno se inspiró y le dio una parte de sí mismo y nos motivó a que de una forma creativa dejáramos una reflexión, una moraleja al final para cautivar a los que nos leyeran”. Una estudiante dijo sobre taller de PowToon "yo era feliz con los muñequitos y me fue más fácil entender el tema de APA”. La educación para el siglo actual ha de ser integral y toda acción dirigida a las personas debe tener su enfoque humanístico.

Finalmente, se les preguntó a los estudiantes por una nueva denominación del curso y surgieron las siguientes propuestas: Aplicaciones TIC, Tecnología Integrada, Liderazgo Tecnológico, Tecnología e Informática, Liderazgo a través de las TIC

Debe contener el impacto, forma de evaluar dicho impacto y resultados.

\section{CONCLUSIONES}

Los resultados del piloto, fueron presentados en los comités curriculares de los programas de la Facultad, motivando a los directores de los programas y profesores a vincularse y seguir las redes sociales del curso para verificar los "productos" y difundirlos a toda la comunidad educativa. Se acordó por votación unánime que el curso cambie de denominación a “TIC aplicada a los Negocios”, acogiendo en parte la propuesta de los estudiantes y eliminando la palabra informática de la denominación dado que es limitante e incluye concepto de programación y redes que nunca han sido objetivo del curso.

En el segundo semestre de 2017 se implementará el curso rediseñado involucrando a todos los estudiantes de la facultad, dado que fue refrendado dentro de la reforma curricular institucional en este primer semestre de 2017 y se espera en el 2018 ofrecerlo como curso electivo para las otras facultades de la Universidad.

Como consideración clave presentada en los comités curriculares, se dejó consignado que para la masificación del curso se requiere una formación previa de los docentes en las herramientas desarrolladas en el curso; igualmente, para su mejora continua se recomienda implementar semestralmente herramientas y aplicaciones que estén en la frontera del avance tecnológico para la producción de contenidos, manteniendo el tema de la ética y valores de manera trasversal.

\section{REFERENCIAS}

Elliot, J. (1983). A Curriculum for the Study of Human Affairs: the contribution of Lawrence Stenhouse. http://dx.doi.org/10.1080/0022027830150202

Gibbons M. (1998). Pertinencia de la educación superior en el siglo XXI. Conferencia Mundial sobre la Educación Superior de la UNESCO. Recuperado de: http://www.fceia.unr.edu.ar/geii/maestria/2013/ADOLF O\%20STUBRIN/BIBLIOGRAF\%C3\%8DA\%202013/L ectura\%205.\%20Pertinencia\%20de\%20la\%20educacion \%20superior\%20en\%20el\%20siglo\%20XXI.pdf

Habermas, J. (1986). Conocimiento e interés. Madrid, España: Taurus.

KPMG International Corporative (2014). Future State 2030: The global megatrends shaping governments. Recuperado https://assets.kpmg.com/content/dam/kpmg/pdf/2014/02/ future-state-2030-v3.pdf

Ley No. 749. Diario oficial de la República de Colombia, Bogotá, Colombia, 19 de julio de 2002.

Malagon, L. (2004). Universidad y sociedad. Pertinencia y educación superior. Bogotá, Colombia: Ed. Alma Mater Magisterio.

Ministerio de Educación de Colombia MEN. (2006). Visión 2019: Educación en Colombia. Recuperado de: http://www.mineducacion.gov.co/cvn/1665/articles110603_archivo_pdf.pdf

Posner, G. (2000). Análisis del currículo. Ed. Mac Graw Hill.

Universidad Tecnológica de Bolívar (2005). Proyecto Educativo Institucional PEI. Recuperado de: http://www.unitecnologica.edu.co/sites/web.unitecnolog ica.edu.co/files/descargas/PEI-Diciembre-2005_0.pdf

Zabalza, M. (1997). Diseño y Desarrollo Curricular. Madrid, España: Ed. Morata. 